Die Umwelt und das Problem der europäischen Harmonisierung der Politiken

\section{Kontroverse Welten und Konventionen}

\author{
Umweltpolitik in der realen Welt ist durch vielfache Unsicherheiten gekenn- \\ zeichnet. Für den Umgang damit spielen grundlegende Konventionen eine \\ entscheidende Rolle. Sie entstehen in einem sozialen Prozeß und prägen die \\ nationalen Politiken bzw. Regime entscheidend. Wählt man die vor allem in \\ Frankreich verbreitete Ökonomie der Konventionen sowie das Regimekonzept \\ als Ausgangspunkte der Analyse, so erschließt sich auch das Problem der \\ europäischen Harmonisierung aus einem neuen und fruchtbaren Blickwinkel.
}

$\mathrm{D}$

Von Olivier Godard zehnten im Rahmen von Gleichgewichtsund Pareto-Effizienzmodellen entwickelt wird, schlägt eine Darstellung der Umweltprobleme und deren möglicher Lösungen vor, die der Vorstellung verfestigter Welten entspricht. In diesen sind die derzeitigen bzw. potentiellen Schäden aus wissenschaftlicher Sicht gut bekannt und reversibel und werden durch die Akteure direkt wahrgenommen, wobei ausschließlich die Präferenzen der gegenwärtigen Akteure direkt eine Rolle spielen. Bei staatlichen Politiken geht es darum, die externen Effekte in das ökonomische Kalkül einzubeziehen und die ökonomische Effizienz der politischen Verfahren und Instrumente zu analysieren, um sich in der Praxis so gut wie möglich dem berechneten Optimum zu nähern.

Die positive Analyse der Verfahren, mit denen Umweltpolitiken auf nationaler und gemeinschaftlicher Ebene gewählt werden, zeigt eine ganz andere Realität: Diese Differenzen sind an mehreren Punkten festzustellen:

- Bei der Gewichtung der Ziele mit zunehmender Bezugnahme auf den Begriff der nachhaltigen Entwicklung, ein Begriff, in dem sich sehr unterschiedliche und miteinander rivalisierende theoretische Konzeptionen widerspiegeln (1);

- der Gewichtung der Einschätzungen von Sachverständigen, wobei die Beurteilungen von Naturwissenschaftlern und Ingenieuren gegenüber denen von Wirtschaftsfachleuten eine klar dominierende Rolle spielen, und

- bei der Gewichtung der eingesetzten Instrumente, wobei eine starke Präferenz für Normierungen und reglementierende gegenüber ökonomischen Instrumenten auszumachen ist.
Wenn man das gesamte Ausmaß an wissenschaftlichen Unsicherheiten und Kontroversen, die in Umweltfragen nahezu jeden Handlungsbereich berühren, erfaßt, sind die Differenzen über den oberflächlichen Konsens der Notwendigkeit von Umweltschutz hinaus auch an den Grundfesten dieser Politiken auszumachen. Die wichtigsten Politiken haben sich somit in Kontexten entwickelt, für die hier die Bezeichnung „,kontroverse Welten" vorgeschlagen wird. Diese Art von Kontexten führt zu einer wesentlichen Rolle von Basiskonventionen als Grundlage für die Durchführung von Handlungen. Für die zwischen den einzelnen Ländern feststellbaren Unterschiede im politischen Umgang mit identischen Grundproblemen - wie Müllentsorgung oder Kampf gegen Wasserverschmutzung - hat die Wahl dieser Konventionen, die im Lichte der den europäischen Ländern eigenen Kontexte und der jeweiligen Umweltprobleme zu verstehen ist, eine große Bedeutung.

\section{Kontroverse Welten}

In der kontroversen Welt (vgl. Abb. 1) ist die Wissenschaftlergemeinde leidlich in der Lage, Eventualitäten zu formulieren, um mögliche Verläufe der Entwicklung zu prognostizieren, sie ist jedoch nicht in der Lage, die Phänomene, ihre Ursachen und Konsequenzen in dauerhafter und empirisch belegter Form darzustellen und objektive Wahrscheinlichkeiten anzugeben. Wissenschaftler verschiedener Disziplinen tragen unterschiedliche Hypothesen, Erklärungs- und Prognosemodelle vor, bei denen ganz unterschiedliche mögliche Welten zum Vorschein kommen: Es besteht wissenschaftliche Kontroverse.

Das Bestehen wissenschaftlicher Kontroversen ist ein normales Phänomen der wissenschaftli- chen Entwicklung. In einer kontroversen Welt nähren diese wissenschaftlichen Kontroversen jedoch soziale Kontroversen oder stoßen auf diese, wobei Interessen berührt werden, die eine politische Dimension haben oder kollektive Verantwortungen auf den Plan rufen.

Die Bandbreite möglicher Konsequenzen und die Vermutung ihrer Unumkehrbarkeit führt einige Akteure zu der Meinung, daß sofort gehandelt werden müsse. Andere, für die Notwendigkeit einer rationalen Herangehensweise plädierende Gruppen fordern, solange $z u$ warten, bis stabile Erkenntnisse vorliegen, um ,in Kenntnis der Ursachen" handeln zu können. Die soziale Kontroverse wirkt wiederum auf die wissenschaftliche Kontroverse, da die wichtigsten Akteure (Regierungen, Industriewelt, Nichtregierungsorganisationen) bestrebt sind, ihr strategisches Aktionsfeld und ihren Raum für die gegenseitige Konfrontation auf die wissenschaftlichen Darstellungen der Welt auszudehnen. Entweder beabsichtigen sie damit, die Kontroverse durch das Aufzwingen einer gegebenen ,Wahrheit" vorzeitig abzuschließen oder aber das Gegenteil: Sehr häufig wird das Argument der wissenschaftlichen Unsicherheit vorgebracht, gepflegt oder gar selbst erzeugt, um jegliche Handlungen auf später zu verschieben.

\section{Die Herausbildung von Konventionen}

Die wissenschaftlichen Schlußfolgerungen und Behauptungen werden durch die Strategien der Akteure und bisweilen der Wissenschaftler selbst

Abb. 1: Merkmale der kontroversen Welt (2)

1. Die wissenschaftliche und soziole Konstruktion des Problems dominiert über die direkte Wahrnehmung durch die Akteure (sozial mediatisierte Externalitöten).

2. Die Frage der getrennten Darstellung der Interessen von Dritten ist ebenso wie die Frage der zu berücksichtigenden kollektiven Interessen explizit Teil der Diskussion.

3. Die wissenschafflichen Erkenntnisse bezüglich der für die Planung von Handlungen wesentlichen Aspekte des Problems bleiben daverhaft umstritten. 4. Der Einsatz, um den es bei diesem Problem geht (z.B. kollektive Sicherheit bzw. Überleben der Menschheit), wird zumindest von einigen Akteuren als so kapital angesehen, dof nach ihrer Ansicht angesichts der Gefahr einer unumkehrbaren Schädigung auch ohne wissenschaftlich erwiesene Erkenntnisse unmittelbar gehandelt werden muß. 
instrumentalisiert. Der ökonomische Produktund Technologiewettbewerb wird von einem Wettbewerb zwischen den „Weltsichten" und „Zukunftsvisionen" begleitet, in dem sich epistemische Gemeinschaften bilden und grundlegende, für die Organisation von Handlungen bestimmte Umweltkonventionen festgesetzt werden.

Durch staatliches Handeln wird per Konvention eine bestimmte „Weltsicht“ festgelegt und diese „Weltsicht" auf Dauer in juristischen Bestimmungen, technischen Normen, Haushaltsentscheidungen und der Schaffung von Institutionen festgeschrieben.

Die von der öffentlichen Hand durchgefïhrten Handlungen werden auf der Grundlage dieser Konventionen zur Umgestaltung der die Wirtschaftswelt bestimmenden Institutionen und Vorschriften führen. Es gibt diesbezüglich zwei Schlüsselfelder:

- die technologischen Konventionen (technische Bestimmungen und Normen usw.), die technische Ziele nach ihrer sozialen Zulässigkeit filtern, und

- die Anreizsysteme für Akteure zu einem bestimmten Umgang mit dieser Objektwelt (Information, Preismechanismen, Regulierungsinstrumente)

Das Ergebnis des Wettbewerbs zur Festlegung von Konventionen und Bestimmungen bedeutet letztendlich eine indirekte Abstimmung über die wissenschaftliche Kontroverse und technologische Entwicklung aus ökonomischen und politischen Gründen.

Berïcksichtigt man den Anteil von Willkür, den das staatliche Handeln bezüglich seines Gegenstandes sowie des Zeitpunktes, zu dem es erfolgt, enthält, stellt es ex ante für bestimmte Wirtschaftskräfte eine Bedrohung und für andere Kreise eine strategische Möglichkeit dar. Denn von der „Weltsicht“, die bei Umweltfragen letzten Endes dominieren wird, hängt ebenfalls die Art und Weise $\mathrm{ab}$, in der die institutionellen und technologischen Rahmenbedingungen zur Strukturierung der Märkte organisiert werden. An diesem Punkt kann man ein erneutes Umschwenken im sozialen Prozeß beobachten. Für alle Arten von Akteuren führen die zur Lösung von Umweltproblemen anvisierten Maßnahmen nunmehr zu einer Wahrnehmung des Umweltproblems aus der Perspektive, indirekte Bedrohung/Möglichkeit“. Es gibt jedoch einen Moment, wo die Akteure unterschiedlicher Interessengemeinschaften im Wunsch nach einer Ent- scheidung übereinstimmen. Diese Entscheidung wird häufig auf der in der kontroversen Welt am solidesten erscheinenden Basis durchgeführt, nämlich mit Technologien, die sich bereits im operativen Zustand befinden oder größte Chancen haben, schnell in diesen Zustand zu gelangen. Das heißt, daß diese Technologien von den Netzen der ökonomischen Akteure bzw. gut formierter Organisationen, deren Interessen mit der Entwicklung dieser Technologien in Zusammenhang stehen, getragen werden.

\section{- Soziale Eigendynamik}

Der soziale Prozeß erlangt demnach gegenüber der sich weiterentwickelnden Dynamik der wissenschaftlichen Kontroverse eine Eigenständigkeit, durch die es schwierig werden wird, einmal gefundene Kompromisse, selbst wenn diese einer durch die spätere wissenschaftliche Entwicklung nicht bestätigten ,Weltsicht" entsprechen, erneut in Frage zu stellen: Wurde den rahmenbildenden Investitionen, die in der Schaffung von Strukturen kostenaufwendig sind, einmal zugestimmt, werden diese Investitionen nicht so leicht wieder aufgegeben. Die Verantwortichen, die sich im Rahmen einer solchen Aktion eingesetzt haben, haben damit auch ihr politisches Gewicht eingebracht und sind nicht bereit, dieses zu vergeuden. Die sich an einer neuen Konvention ausrichtende Neustrukturierung von Strategien staatlicher und ökonomischer Akteure macht deren spätere Aufgabe teuer und schwierig.

Für die ökonomischen Akteure stellt sich das Entscheidungsproblem somit in doppelter Hinsicht: Im allgemeinen muß jeder, sofern seine Tätigkeit davon abhängt, versuchen, Prognosen über die sich schließlich durchsetzenden biophysikalischen Entwicklungen der Welt zu machen, und gleichzeitig zu antizipieren, welche „Weltsicht" unter der Wirkung einer sozialen Konvention dominieren wird. Für alle Akteure, die vom direkten Umweltproblem nur am Rande betroffen sind, ist meistens dieser zweite Aspekt ausschlaggebend. Überraschenderweise besteht daherfür die meisten Akteure das Problem weniger in der Antizipation des ,wahren“ Zustands als in der Antizipation der sich sozial durchsetzenden „Weltsicht“, wobei diese von den durch die Führungskräfte auf den Weg gebrachten Strategien abhängt.

Dieses Problem der Antizipation der Antizipationen der anderen im kontroversen Kontext kann im Rahmen eines ausschließlich auf Prognose und Optimierungsrechnung beruhen- dem Ansatz aufgrund der unendlichen Regression zu keiner rationalen Lösung führen. Es ist aber für viele Akteure aufgrund der Befürchtung, daß die kollektive Antizipation sich auf eine spontane, weitgehend suboptimale Konvention fokussiert, ein starker Anreiz, an einem Verhandlungsprozeß über eine bestimmte Konvention teilzunehmen. Auf welchen Variablen jedoch sollte man die Konvention aufbauen? Die Gefahr-der voreiligen Festlegung einer „Weltsicht" bzw. die Entscheidung für bestimmte Technologien mit lock-in-Phänomenen bei schlechteren Technologien vom „End-of-Pipe“-Typ (3) legt einen anderen Weg nahe: Die zur Gewährleistung einer ausreichenden ökonomischen Vorhersehbarkeit erforderliche Konvention sollte auf einem Regime politischer Instrumente und auf Verfahrensregelungen (Abstimmung mit den jeweiligen Vertretern, Zeitplan für fortwährende Überprïfungen der Politik, ...) aufbauen.

\section{Regimebildung}

Insgesamt kann der Prozeß der Erarbeitung von Politiken als ein Handlungssystem gesehen werden, welches sich anhand von Überzeugungen, Prognosen und Wahrnehmungen, die sich zusammen mit den Strategien von Akteuren bilden, sich diesen überlagern und schließlich durch die Definition von Regeln in Institutionen gefestigt werden, selbst organisiert. Dieser Prozeß unterstreicht die Abhängigkeit kollektiver Entscheidungen von Grundkonventionen, also ursprünglichen kognitiven, normativen und institutionellen „Matrizen“, die je nach Land, Tätigkeitsbereich und Zeitraum unterschiedlich sind. Es muß zwischen den festgelegten Zielen, angewandten Instrumenten und dem institutionellen, ökonomischen und vertraglichen Kontext, in dem die technischen und organisatorischen Entscheidungen getroffen werden, jedoch Kohärenz bestehen. Genau dies will das Regimekonzept deutlich machen.

Länder, die wie die europäischen Länder bezüglich ihres ökonomischen Entwicklungsniveaus miteinander vergleichbar sind und sich auch kulturell nahe stehen, haben in ihren Grundannahmen und Mechanismen sehr unterschiedliche Regime für den Umgang mit Umweltproblemen entwickelt, um dieselben Probleme wie z.B. die Abfallbewirtschaftung zu lösen. Wie interagieren diese Regime, welche Notwendigkeiten bestehen für eine Koordinierung, wie ist ihre Koexistenz zu organisieren? 


\section{Das Beispiel Abfallwirtschaft}

Die Vision, die man für die Organisation einer zukunftsfähigen Art des Umgangs mit Abfällen entwickeln kann, hängt offensichtlich von den Grundtypen der anvisierbaren technischen Lösungen ab. Sie ist jedoch nicht von einer allgemeineren Vorstellung vom materiellen Funktionieren der Wirtschaft und ihrem Einfluß auf die Wahrung der allgemeinen physikalischen und biologischen Gleichgewichte zu trennen. Unter Betrachtung beider Aspekte kann man drei aktuelle, rivalisierende „Mythen“ in Europa ausmachen, welche die eingeschlagenen Politiken umfassen, wobei eine jede einen anderen Weg für die Abfallbewirtschaftung privilegiert (4):

1. Der Mythos der „Beherrschung und Eingrenzung von Schadstoffströmen", der annimmt daß die Festsetzung solcher Ströme und die Verhinderung ihrer Zirkulation in der Natur mittels Deponierung bzw. Verbrennung möglich ist;

2. der Mythos der ,ewigen materiellen Kreisläufe", durch den die Vorstellung von Wirtschaftswachstum ohne signifikante Auswirkung auf die Umwelt mit der Vision einer Recycling-Gesellschaft aufrechterhalten werden kann und

3. der Mythos der ,dematerialisierten postmodernen Gesellschaft", bei der davon ausgegangen wird, daß eine Reduzierung der für die Produktion und die Aufrechterhaltung modernen Lebens erforderlichen Stoff- und Energieströme durch Prävention von der Wurzel an in großem Ausmaß möglich sei.

Diese „Mythen“ könnte man auch als Utopien bezeichnen. Sie stellen stark eingeschränkte Zustände dar, die einzeln genommen als Organisationsprinzip in der realen Welt nicht lebbar wären. Sie stellen nichtsdestotrotz ein Hilfsmittel dar, welches der konkreten Transformation von Regulierungsmodi Sinn verleiht.

In der Tat stïtzen sich die drei Mythen in einer Kreisbeziehung gegenseitig, da ein jeder seine Lösungen dort einbringt, wo die anderen beiden Lösungen Lücken aufweisen. Das, was wir heute industrielle Ökologie nennen (5), ist der zeitgenössische Ausdruck einer zusammengesetzten Utopie, welche die drei genannten Mythen in einer einheitlichen, alle Widersprïche ausblendenden Konzeption vereint.

Auf diesen konventionellen Fundamenten sind die verschiedenen nationalen Regime der Abfallbewirtschaftung in Europa aufgebaut.

Worin besteht das Problem einer nicht koordinierten Entwicklung von nationalen Regimen? Ist es nicht besser, sich zunächst auf das Subsidiaritätsprinzip zu beziehen? Warum sollte man die Umweltpolitiken auf europäischer Ebene koordinieren? Die Gründe liegen darin, daß die strukturellen Unterschiede der nationalen Regime transnationale Auswirkungen haben: Beispielsweise wurde der Marktzugang von Unternehmen, die Getränke exportieren, verändert; Störungen in den Recycling-Unternehmen der Nachbarländer waren die Folge. Die Probleme machten ebenfalls deutlich, wie wichtig der Prozeß der Verbreitung von Normen zwischen den Iändern ist. Schließlich wird auch das Problem des Ausgleichs zwischen unterschiedlichen, von der Europäischen Union verfolgten Zielen und besonders zwischen Binnenmarkt und Sicherung von Gesundheit und Umwelt in den Vordergrund gerïckt.

\section{Zwei europäische Szenarien}

Am Anfang der Europäischen Gemeinschaft stand die Überzeugung, daß sich Europa dank der Schaffung eines gemeinsamen, auf Freihandel innerhalb der Gemeinschaft basierenden Marktes entwickeln würde. Mit dem Aufkommen der Umweltproblematik in der Öffentlichkeit, insbesondere in den Ländern Nordeuropas, als eines der markantesten Phänomene der 70er und 80er Jahre wurden auf nationaler und regionaler Gesetzgebungsebene zunehmend Reglementierungen eingeführt. Diese haben zu diversen Einschränkungen geführt, deren objektive Wirkung in einem Abbremsen des innergemeinschaftlichen Freihandels bzw. dem Ausschluß bestimmter Güterarten aufgrund anderer Prinzipien bestand. Über die Umweltproblematik findet eine neue Form des Konflikts zwischen Markt und Region statt. Mit der formalen Anerkennung der Umwelt als Kompetenzbereich der Gemeinschaft hat sich dieser Konflikt seit den Verträgen von Maastricht und Amsterdam institutionalisiert, wobei die weitere Entwicklung auf zweierlei Wegen möglich ist.

Der erste mögliche Weg: Der europäischen Instanz gelingt es, den durch die Mitgliedsstaaten aufgestellten Regulierungsrahmen so zu harmonisieren, daß Umweltschutz auf einem hohen Qualitätsniveau mit dem freien Warenaustausch in Einklang gebracht werden kann. Eine zunehmende Standardisierung und Normierung für Produkte und Techniken sowie die Erarbeitung von akzeptierten Äquivalenzsystemen wären Triebfedern dafür. Diese Option entspricht deutlich einem hohen politischen Integrationsniveau auf europäischer Ebene sowie der Verfolgung des Ziels eines einheitlichen Marktes als prioritäres Ziel der Union.

Der zweite mögliche Weg: Das Prinzip des freien Warenverkehrs verliert seinen allgemeingültigen Wert und wird auf Güter beschränkt, deren Eigenschaften keine Beeinträchtigung von Gesundheit und Umweltqualität darstellen. Die Frage der Risiken steht hier an erster Stelle. Strategische Prinzipien werden ausgearbeitet, um sich den Risiken zu stellen. Durch die Mobilisierung des inzwischen in das gemeinschaftliche Recht aufgenommenen Vorsichtsprinzips (6) kann man bei der Regulierung der wirtschaftlichen Austauschkreisläufe für eine ganze Reihe von „besonderen“ Gütern selektiv zu einer Politik auf regionaler Ebene zurïckkehren. Das Fundament dafür wäre die Unfähigkeit eines auf europäischer Ebene integrierten Systems, die sanitäre Sicherheit und Umweltqualität der in lange Austauschkreisläufe eingebundenen Produkte zu garantieren. Die Abfallbewirtschaftung, unabhängig davon, ob es sich um Haus- oder Gewerbemüll handelt, ist für die eben beschriebenen Spannungen exemplarisch.

\section{Anmerkungen}

(1) Godard, 0.: Le développement durable. Paysage intellectuel. In: Natures-Sciences-Sociétés, Vol. 2 (1994), (4), octobre, pp. 309-322.

(2) Godard, 0.: Social decision-making under conditions of scientific controversy, expertise and the precautionary principle. In C. Jörges, K.H. Ladeur and E. Vos (eds.): Integrating scientific expertise into regulatory decision-making National traditions and European innovations. Nomos, Baden-Baden 1996, pp. 39-73.

(3) Kemp, R., L. Soete: The greening of technological progress. An evolutionary perspective. In: Futures, Vol. 24 (1992), (5), June.

(4) Buclet N., 0. Godard: Municipal waste management in Europe. A comparison of national regimes. In Buclet N., 0 . Godard (eds.): Municipal Waste Management in Europe - A Comparative Study in Regime-building. Kluwer Academics Publ., Dordrecht \& London 1999, forthcoming.

(5) Erkman, S.: Vers une écologie industrielle. Editions Charles Léopold Mayer, Paris 1998.

(6) Godard, 0. (ed.): Le principe de précaution dans la conduite des affaires humaines. Ed. de la Maison des sciences de l'homme et INRA-Editions, Paris 1997.

\section{Der Autor}

Dr. Olivier Godard ist Forschungsdirektor am Centre National de Recherche Scientifique.

Kontrakt: CNRS, Laboratoire d'Economètrie, Ecole Polytechnique, 1 Rue Descartes, F-75005 Paris. 
(c) 20I0 Authors; licensee IÖW and oekom verlag. This is an article distributed under the terms of the Creative Commons Attribution Non-Commercial No Derivates License (http://creativecommons.org/licenses/by-nc-nd/3.o/), which permits unrestricted use, distribution, and reproduction in any medium, provided the original work is properly cited. 